\title{
Traumatisches Colobom des Pigmentepithels der Iris.
}

\author{
Von \\ Dr. Th. Gelpke, \\ Augenarzt in Karlsruhe.
}

Im Anschluss an die in Band XXXII. 2. und XXXIII. 1. dieses Archivs von Franke theils gesammelten, theils selbst beobachteten Fälle von ungewöhnlichen Verletzungen der Iris müchte ich über eine andere eigenartige Irisläsion berichten, die ich im Laufe dieses Jahres zu beobachten Gelegenheit hatte. Es schien mir dieselbe besonders deswegen der Mittheilung werth, weil in der-mir wenigstens zugänglichen - Literatur keine ähnliche verzeichnet ist. Der Fall war folgender:

Am 8. Mai d. J. wurde dem 16jährigen Feilenhauer Ph. Burkard aus Pforzheim von einem Kameraden das untere Ende einer Feile aus einer Entfernung von ca. 3 Meter gegen das linke Ange geschleudert. Das Corpus delicti hatte ungefähr die Form einer vierseitigen Pyramide, eine Länge ron $5 \mathrm{ctm}$ und war an dem einen Ende scharf zugespitzt.

Patient empfand im. Moment der Verletzung heftigen Schmerz im getroffenen Ange und hatte die Empfindung, als ob etwas Flüssigkeit aus dem Auge hervorquelle. Eine Röthung und Verletzung der Lider war nicht vorhanden. Das Sehvermögen des verletzten Auges sank sofort, doch konnte Patient mit dem Auge noch deutlich seine nächste Umgebung erkennen. Patient be- 
gab sich gleich nach dem Unfall zu einem Arzt, der Atropin einträufelte und einen Verband anlegte. Die anfänglich heftigen Schmerzen cessirten darauf. Am nächsten Tage bemerkte jedoch Patient, dass das Sehvermögen des verletzten Auges so bedeutend abgenommen hatte, dass nur Handbewegungen in ca. 1 Meter Entfernung wahrgenommen werden konnten. Auf spätere Nachfragen erfuhr ich nur soviel, dass ansser der Hornhautverlotzung: nichts weiter als eine Verengerung der Pupille - kein Hyphaema oder sonstige sichtbare Veränderung damals zn bemerken gewesen. Am dritten Tage nach der Verletzung traten trotz Atropin und Verband wieder heftige, bohrende Schmerzen im linken Ange ein, woranf der Patient zu weiterer Behandlung mir zugewiesen wurde.

Der Status praesens am 12. Mai (demnach 4 Tage nach der Verletzung) war folgender:

In der Umgebung beider Augen keine Veränderungen, spee. keine Spuren einer voransgegangenen Verletzung.

Das rechte Auge sammt seinen Adnexen völlig normal. SR. $=6 / s$. - Links Lider nieht geschwollen, die Randtheile leicht geröthet.

Conjunctiva palp. mässig stark injieirt, im Fornix leicht geschwollen. Kein Secret anf derselben.

Die Gefässe der Conjunctiva bulbi stärker als in der Norm gefullt. Keine Chemosis.

Um die Hornhant eine feine violette, episclerale Injection, die besonders nach oben-innen sehr ausgeprägt ist.

Hornhaut in den peripheren Theilen klar und durehsichtig, im Centrum leicht difus getrubt. Etwa $1 \mathrm{~mm}$ vom Hornhautscheitel nasalwärts eine vertikale $3 \mathrm{~mm}$ lange, scharf berandete, verklebte $W$ unde, die schräg nach dem nasalen Pupillenrand hin, das ganze Hornhautparemchym durchsetzte. Zwischen den vorderen Lefzen der ersteren ein eitriger necrotischer Gewebsfetzen, der sich nur unvollständig mit der Pincette entfernen liess. - Von der inneren Wundoffnung ein gelbröthliches, gezacktes feines Fädchen schräg nach oben-innen bis, uud noch unter den betreffenden Theil des Pupillenrandes der Iris sich hinziehend (Synechia anterior) woselbst dasselbe an der vorderen Kapsel angeheftet war.

Vordere Kammer, oben-innen tiefer, als aussen-unten.

Kammerwasser klar, ohne pathologische Beimengungen (Blut oder Eiter). 
Iris hyperämisch, bei direct auffallendem licht in ihrer Structur nicht verändert.

Pupille mittelweit, enger, als rechts, rund. Reaction minimal, nach aussen - unten am ergiebigsten. Pupillarrand nirgends adhärent, jedoch an der Stelle, wo er die oben erwähnte Synechie tangirt, leicht ausgefranzt, seines Pigmentsaumes beranbt und durch die Synechie etwas von der vorderen Linsenfläche abgehoben.

Sphincterpartie völlig intact.

Liess man nun focales Licht, so seitlich wie möglich, auf die Tris fallen, und zwar so, dass die Spitze des durch die Linse gesammelten Lichthegels etwa die Anheftungsstelle der Synechie an der Linse traf, so sah man durch die betreffende Partie des Irisgewebes eine mässig hell erlenchtete ca. $2 \mathrm{~mm}$ breite Spalte hindurchschimmern, die in der Richtung der mehrfach erwähnten Synechie (als. radiär) bis zur Giliarinsertion der Iris - hinter der Iris - verlief. Die Ränder dieser Spalte waren ganz scharf, gradlinig und verliefen fast parallel zu einander. Wenn man dann ferner mit dem Spiegel directes Licht auf diese Irispartie fallen liess, so erhielt man - auffallender Weise - keinen röthlichen Reflex, wie a priori erwartet wurde. Die Ursache lag der Hauptsache nach in der Linse.

Erweiterte man nämlich die Pupille durch Atropin - was übrigens nur unvollständig und in geringem Grade gelang so sah man zunächst im oberen inneren Quadrant der Linse eine unregelmässig viereckige Narbe der vorderen Kapsel, in welche die vordere Synechie eingefügt war. Von dieser Narbe breitete sich eine flächenhafte Trübung der angrenzenden rorderen Corticalis aus, die ihrerseits sich durch die ganze Dicke der Linse hindurch bis zum hinteren Cortex erstreckte. Die cataractöse Trübung reichte bis in das Pupillargebiet hinein; ihre Begrenzung nach dem Aequator hin konnte jedoch wegen der unvollständigen Mydriasis nicht verfolgt werden. - Die Linse in toto stand ferner deutlich schräg und war ron hintenoben nach vorn-aussen luxirt.

Ein rother Reflex aus dem Innern des Auges konnte durch die cataractöse Trübung überhaupt nicht, nach aussen oben und unten nur sehr schwach wahrgenommen werden. Folglich war es auch nicht möglich, die oben erwähnte Spalte, die der cataractösen Trübung in ihrer Lage entsprach, durch direct 
auf dieselbe fallendes Licht $z$ arleuchten resp. als solehe dureh das Irisstroma hindurch a erkennen.

Einzelheiten vom Fundus waren absolut nicht erkennbar. $\mathrm{T}-2, \mathrm{~S}=$ schwach Finger in nächster Nähe erkannt. Projection richtig. Bulbus auf Druck etwas empfindlich.

Die BehandIung bestand in zweistündichen Atropineinträufelungen, zeitweiligen Eisumschlägen, Druckverband und Ruholage.

Während derselben horten gleich in den nächsten Tagen dio anfänglichen Schmerzen völlig auf. Die pericorneale Injection verschwand fast völlig, der necrotische Gewebsfetzen stiess sich aus der Wunde ab, die letztere vernarbte glatt.

Auch $\mathrm{S}$ wurde besser, indem Finger in $1 \mathrm{~m}$ richtig gezählt wurden. Die Verhältnisse an der Iris und Pupille blieben stationär; ebenso die partielle Linsentrübung.

Nach achttägiger Behandlung wurde, da der Bulbus vollig reizfrei war, Atropin ausgesetzt und statt dessen, um ein Sprengen der vorderen Synechie durch den sich contrahirenden Pupillarrand $z u$ bewirken, Eserin eingeträufelt. Hierauf trat eine Verengerung, zugleich aber auch eine unregelmässige Verziehung der Pupille ein, dadurch, dass der Theil des Pupillenrandes, welcher auf der Synechie so zu sagen ritt, sich nicht contrahirte. Schuld komute hieran nicht eine mangelhafte Action des Sphincter iridis sein, denn die betreffende Trispartie war wie oben ausdrücklich erwähnt - vollig intact. Vielleicht bestand eine Flächenverwachsung der Iris mit der Kapselwunde? Jedenfalls konnte aber auch die Synechie selbst im Wege stehen. Ich entschloss mich daher, dieselbe operativ zu entfernen und durchtrennte dieselbe $z$ a dem Behufe mit der Discissionsnadel. Meine Vermuthung traf ein, denn sofort nach der Durchtrennung wurde die Pnpille vollständig rund und folgte der Eserin-Ginwirkung:

Patient wurde dann nach 4 Tagen vorlaufig entlassen.

Vier Wochen später sah ich ihn wieder und konnte einen völlig reizlosen Zustand des Auges constatiren. Eine Veränderung war nur insofern eingetreten, als die Hornhautnarbe sich sehr verschmälert hatte, die Synechie völlig resorbirt und die dem Pigmentcolobom entsprechende Partie des Irisstromas in dentlich schlotternde Bewegung gerathen war. Die Pupille hatte eine vollig runde Gestalt, war eng, reagirte aber immer noch sehr schwach. Dem entsprach eine noch sehr mangellafte 
Traumatisches Colobom des Pigmentepithels der Iris. 163

Lichtempfindung. S betrug nur knapp 1/60. Die Linse zeigte dasselbe Bild bezüglich ihrer Stellung und ihres Aussehens. Einzelheiten vom Fundus konnten immer noch micht erkannt werden.

Um in kurzen Zügen die obigen Veränderungen zu resumiren, so handelte es sich in unserem Fall also um eine Verletzung des Auges durch ein spitzes kantiges Stück Eisen. Dasselbe hatte mit ziemlicher Vehemenz die Hornhaut getroffen, dieselbe perforirt, die Linsenkapsel an einer Stelle zerrissen und dadurch eine Cataracta traumatica circumscripta herbeigeführt. Mit diesen Veränderungen erfolgte dann zugleich eine partielle Zerreissung des Pigmentbelags der Iris - die Bildung eines Colobomairidis partiale, eine unvollständige Luxation der Linse und wahrscheinlich eine Blutung in den Glaskörper durch die Ruptur von Ciliarkörpergefüssen. Nachdem der Fremdkörper dann das Auge verlassen hatte, legte sich ein durch die Eisenspitze losgelöstes und an der Linsenkapsel fixirtes Fäserchen des verletzten Pupillarrandes in die Perforationsofffnung der Hornhant und wurde dann mit der Wiederherstellung und Vertiefung der vorderen Kammer gedehnt und in die Länge gezogen.

Was die specielle Genese des Pigmentepithel-Coloboms - denn dieses interessirt uns ja hauptsächlich in unserem Fall - anbetrifft, so wären zwei Entstehungsarten denkbar.

Einmal könnte durch das Aufprallen des Eisenstüeks auf die Hornhaut eine Einknickung des inneren-oberen Irisquadranten nach hinten stattgefunden haben und dadurch indirect ein Riss des Pigmentbelags entstanden sein. Zu Gunsten dieser Annahme spräche die bekannte histologische Thatsache, dass der hintere Pigmentbelag der Iris sehr unelastisch ist und daher mechanischen Einwirkungen, denen das Irisstroma energischen Widerstand entgegensetzt, unterliegt, d. h. zerreisst. 
Die begleitenden Nebenverletzungen - nämlich die glatte Hornhautwunde, die eigenartige Verletzung der Tinsenkapsel - lassen jedoch die Entstehung causa directa für plausibeler erscheinen. Darnach wäre mit grösster Wahrscheinlichkeit das Eisenstück mit dem spitzen Ende in die Hornhaut eingedrungen, dann schräg nach innen-oben, zwischen Pupillarrand and vorderer Linsenkapsel bis zur Ciliarinsertion der Iris weiter vorgedrungen, indem es dabei den betreffenden Theil des Pigmentbelags vor sich herschob, sowie die Linsenkapsel eroffnete, und dann, seine Propulsivkraft verlierend, wieder aus dem Auge herausgefallen. 